\title{
Is ultrasound-guided thoracic paravertebral nerve block better than intercostal nerve block for video-assisted thoracic surgery under spontaneous-ventilating anesthesia?
}

DDaoyun Lei ${ }^{1}$

(iD) Yeqin Sha ${ }^{2}$

DLianping $\mathrm{He}^{2}$

1. Jiangsu University, Jiangsu, Zhenjiang 212013, China. 2. Department of Immunology, Nanjing medical university, Nanjing, Jiangsu 211166, China.

Daoyun Lei and Yeqin Sha contributed to this work.

Dear Editor,

We read with great interest the study by Zheng et al. ${ }^{1}$. It revealed that patients under ultrasound-guided thoracic paravertebral nerve block had a lower incidence of severe mediastinal flutter (grade three) during video-assisted thoracic surgery for pulmonary lobectomy compared with those under ultrasound-guided intercostal nerve block. The authors concluded that thoracic paravertebral nerve block was better than intercostal nerve block during video-assisted thoracic surgery for pulmonary lobectomy. This is of great significance in the selection of techniques for blocking chest nerves during thoracic surgery. However, in my opinion, more factors should be taken into consideration for drawing this conclusion.

Firstly, only some patients are suitable for spontaneous-ventilating anesthesia. Therefore, the authors should confirm the types of thoracoscopic surgery. Additionally, no relevant data indicated the specific types of thoracoscopic surgery. The postoperative analgesic effect in different anesthesia approaches was not evaluated. The Visual Analogue Scale (VAS) or other methods should be used to assess the postoperative analgesic effect.

There are several postoperative complications for video-assisted thoracic surgery, such as intensive care stay, total hospital stay, and mortality rate ${ }^{3}$. However, only the incidence of mediastinal flutter was explored in this study. Therefore, more clinical data should be collected to exclude the side effect of ultrasound-guided thoracic paravertebral nerve block and intercostal nerve block so that a conclusion can be reached.

\section{REFERENCES}

1. Zheng Y, Wang H, Ma X, Cheng Z, Cao W, Sgao D. Comparison effect of ultrasound-guided thoracic paravertebral nerve block and intercostal nerve block for video-assisted thoracic surgery under spontaneous-ventilating anesthesia. Rev Assoc Med Bras. 2020;68(4);452-457.

2. Li X, Vigil JM, Stith SS, Brockelman F, Keeling K, Hall B. The effectiveness of self-directed medical cannabis treatment for pain. Complement Ther Med. 2019;46:123-30.

3. Popovici BI, Matei D, lacoban L, Simion I, Man M, Al Hajjar N, et al. The impact of thoracic paravertebral block over post-operatory evolution in open lobectomy. Ann Ital Chir. 2019;90:551-9.

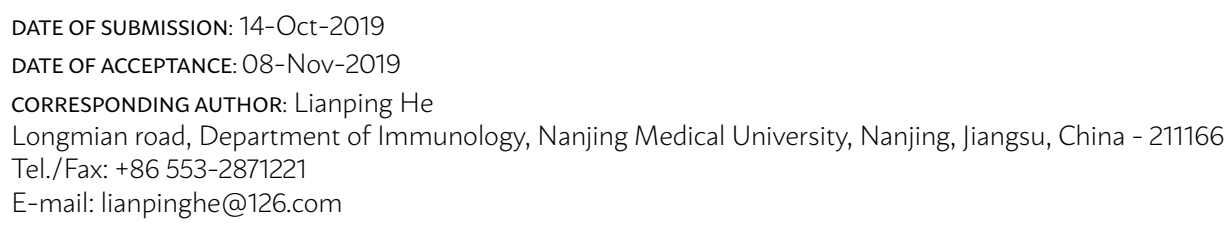

